\title{
High-throughput approaches for genotoxicity testing in drug development: recent advances
}

This article was published in the following Dove Press journal:

International Journal of High Throughput Screening

II January 2016

Number of times this article has been viewed

\author{
Roopasri Ranganatha \\ Sridhara Chakravarthy \\ Sunilkumar Sukumaran \\ Anthem Biosciences Pvt Ltd, \\ Bangalore, India
}

Correspondence: Sunilkumar Sukumaran Anthem Biosciences Pvt Ltd, 49 Canara Bank Road, Bommasandra Industrial Area - Phase I, Bommasandra, Hosur Road, Bangalore, Karnataka 560099, India Tel +918066724000 Fax +918066724020 Email sunilkumar.s@anthembio.com

\begin{abstract}
New molecules targeted at enhancing quality of life undergo various tests to evaluate their efficacy and biosafety before approval. However, there is an urgent need to develop a standardized set of tests and regulatory standards to ensure all molecules comply with biosafety standards at all stages. Though there is a battery of tests used to assess the toxicity of various compounds under study, the inability of these tests to yield a consensus serves as a major drawback. In addition, the time-consuming and labor-intensive nature of these tests does not aid in accelerating the process of screening and identification of molecules with high efficacy and low toxicity. Modernization and standardization of a current battery of tests to a common end point in combination with identification of novel molecular markers for assessing genotoxicity seems to be the way forward. The establishment of high-throughput screening to assess various biomarker parameters, such as DNA damage to evaluate genotoxicity, offers rapid, costeffective, and non-labor-intensive screening, a potential solution to the challenge mentioned. We here review the tests that are currently in use for assessing the genotoxicity potential of various compounds. Furthermore, the review also offers an overview of recent advancements in conventional techniques and use of various molecular markers in an effort to establish highthroughput screening methods that could revolutionize future toxicology studies.
\end{abstract}

Keywords: genotoxicity, biomarkers, high-throughput screening

\section{Introduction}

Toxic substances exhibiting their effect on cell viability are referred to as cytotoxins. Further, classes of substances capable of interacting and damaging the genome within a cell are referred to as genotoxins. Therefore, genotoxicity can be described as the ability of a substance (naturally occurring or chemically synthesized) to cause damage to the genetic information within a cell. This DNA damage could result in mutations, thus promoting carcinogenesis or laying the foundation for congenital disorders. The damage caused by genotoxic agents may involve direct interaction with the DNA, resulting either in base substitutions, frame-shift mutations, or even double-stranded breaks. ${ }^{1}$ In other cases, the genotoxic substances may interact with various proteins that are either involved in replication (mitosis) or maintaining chromosomal stability.

In an era where innumerable biological and synthetic compounds are perpetually introduced into the market every day in various forms, such as pharmaceuticals, cosmetics, nutraceuticals, fertilizers, pesticides, and nanoparticles, to serve multiple purposes, the need to develop rapid screening methods to evaluate their biosafety in addition to their efficacy has gained paramount importance. Several drugs that are currently in use to treat various medical conditions, such as cancer, microbial 
infection, hypertension, depression, and inflammation, have demonstrated genotoxic effects. ${ }^{2-4}$ In addition, other classes of compounds, such as metal nanoparticles, food additives, food preservatives, food flavorings, and commonly used chemicals, such as acetaldehyde, hydroquinone, benzyl alcohol, and benzoic acid, have also been reported to be genotoxic and carcinogenic. ${ }^{5}$

This is of great concern, as a number of in vitro and in vivo tests are in use to assess the safety of various molecules. However, there is no single test that is capable of detecting all genotoxic end points, and thus a combination of in vitro and in vivo analyses needs to be carried out to meet the regulatory requirements. It is also important to note that it could be years before the long-term adverse effects caused by the use of these molecules surface.

In addition, the results obtained through diverse tests can be highly variable, thereby making it very difficult to obtain a consensus. Therefore, having similar end points in the various approaches employed would enable a comparative analysis of the data obtained. The development of a model replicating an animal system yet providing a platform for the effective study of various signaling pathways to assess the mode of action of a compound would undoubtedly aid genotoxicity studies. However, the establishment of simple, rapid, cost effective, and non-labor-intensive high-throughput screening (HTS) techniques to assess the genotoxicity of various molecules intended for medical use at early stages of drug development itself ranks high on the priority list. With the aid of technology, modernization of conventional techniques, along with development of novel techniques to achieve the same, may revolutionize the entire genotoxicity-screening process.

Table 1 highlights the various approaches currently in use for assessing genotoxicity, the advantages and disadvantages of each approach, their ability to be used as a high-throughput test, and their regulatory status.

\section{Prokaryotic systems}

\section{The Ames test (bacterial mutagenesis test)}

The Ames test is one of the most widely used methods to assess genotoxicity. It was developed by Ames et al as a fast and sensitive assay to evaluate the ability of a compound to induce mutations in DNA. ${ }^{6}$ The test has been used to determine the mutagenicity of various compounds, and was first validated using 300 chemicals, most of which were known carcinogens. Since then, the test has undergone additional validations, as a result of which a number of modifications have been incorporated periodically to improve its efficacy.
The ability of a compound to revert the mutation to the wild-type genotype serves as the principle of the Ames test. Bacteria require histidine for synthesizing various proteins, and only those capable of synthesizing histidine will survive. The test involves plating His ${ }^{-}$Salmonella typhimurium onto media containing trace amounts of histidine and adding chemicals to be tested for mutagenicity. Colonies arise only if the compound is capable of reversing the mutation and converts the His ${ }^{-}$to $\mathrm{His}^{+}$. In order to incorporate the aspect of mammalian host metabolism, liver extracts used to be added in the original Ames test, thereby simulating the action of mammalian liver enzymes that are known to play a role in metabolite generation. ${ }^{7}$ A variety of concentrations of each chemical are usually tested to generate a dose-response curve. The analysis of these curves highlights various aspects, including the activity spectrum in tester strains, classification of the chemical as either frame-shift or basesubstitution mutagens, the mutagenic potency (minimum concentration required for exhibiting mutagenicity), and the minimal concentration at which auxotroph growth is inhibited. However, it is important to note that the rate of mutagenicity can vary due to the rate of chemical absorption by cells and differential metabolism of compounds. ${ }^{8}$

In order to facilitate the screening of more compounds in a shorter amount of time, an alternate method known as the "fluctuation method" is employed. ${ }^{9}$ In this approach, the entire experiment is performed in a liquid culture, and the score is obtained by the enumeration of wells that have changed from purple to yellow in a 96-well plate. Though the principle remains the same, the addition of a $\mathrm{pH}$ indicator to the media enables the frequency of the mutation to be counted. The sample is compared to the control in order to evaluate the genotoxicity of the substance. The 96-well plates are incubated for up to 5 days, with counting of the mutated colonies every day and comparison to the basal rate of reverse mutation. Besides being comparable to the traditional Ames test, the fluctuation method also enables testing of elevated concentrations, as well as more chemical compounds while using only small quantities of the compound. It is a simpler and less labor-intensive method, owing to the use of a colorimetric end point, than the traditional Ames test.

Cerep offers a simpler and compact version of the Ames test utilizing liquid media, 384-well plates, and four Salmonella strains: TA98, TA100, TA1535, and TA1537. The results are obtained after 96 hours of incubation of the bacterial strains with the compound through spectrophotometry. Compounds are tested in all four bacterial strains, both in the presence and absence of S9 rat liver homogenate. 


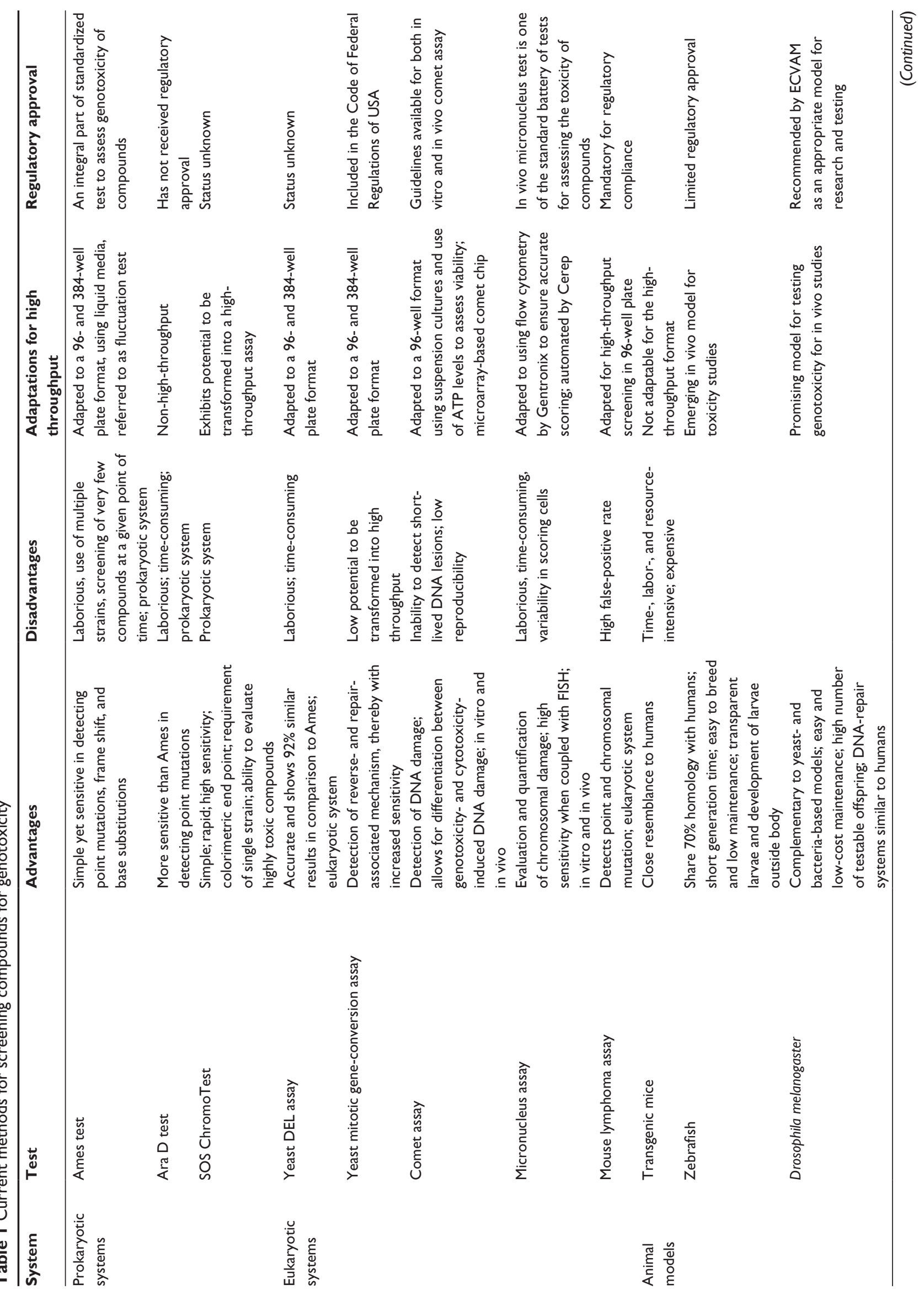


A bacterial cytotoxicity assay is carried out in parallel to rule out false negatives. ${ }^{10}$

Another bacteria-based genotoxicity screen is the Ames MPFTM (microplate format) bacteria-mutation test developed by Xenometrix, which can be used both in the presence and absence of S9 fraction. The auxotrophs are exposed to six doses of the test chemical in a 24-well plate. After a brief exposure period, the cultures are diluted using histidine-free media with a $\mathrm{pH}$ indicator, and the contents of the 24-well plate are transferred into 48 wells of a 384-well microplate. This is followed by a 48 -hour incubation period. During this period, the cells that have mutated back to the wild-type genotype, either spontaneously or as a result of exposure to the test chemical, divide. As a result, cellular metabolism reduces the $\mathrm{pH}$ of the medium, changing the indicator color from purple to yellow. Upon completion of the incubation period, 384-well plates are scored by differentially counting colored wells spectrophotometrically in order to avoid operator bias and obtain highly accurate data. These yellow revertant wells are counted for each dose and compared to the zero dose. ${ }^{11}$ A rapid mutagenicity assay based on the Ames test and ATP bioluminescence technique has also been developed. ${ }^{12}$ Recent advancements to improve further the sensitivity of Ames test involves a biochemical version where two genetically modified strains expressing the luxCDABE operon from Xenorhabdus luminescens are used. The modified Ames test has been validated with 105 compounds that have previously been evaluated in the standard Ames test. ${ }^{13}$

The simplicity and the minimal time requirement serve as major advantages for the high-throughput forms of the Ames test. However, on the other hand, a major drawback lies in the inability to predict mutagenicity/genotoxicity in vivo completely.

\section{The Ara D test}

This test is an alternative assay that detects mutations in the arabinose operon. The strains used for this assay possess a mutation in the $A r a D$ gene, which promotes the accumulation of a toxic substance in the presence of arabinose. Genotoxic substances inactivate the operon, thereby preventing the metabolism of arabinose to toxic intermediates. It is reported to be more sensitive in detecting mutagens capable of inducing point mutations in comparison to the Ames test. ${ }^{14}$ However, the assay has not yet received regulatory approval.

\section{The SOS chromotest}

Another test that utilizes Escherichia coli $\mathrm{K} 12$ to detect DNA-damaging agents is referred to as the SOS chromotest. 
Most of the bacteriological toxicity studies take the response of the cell to the primary action of the agent as the end point. This test utilizes a strain that has been developed with a fused operon system, placing the structural gene of lac $Z$ under the control of the $s f i A$ gene (an SOS function involved in cell-division inhibition) to develop a simple and direct colorimetric assay. ${ }^{15}$ The assay provides quantifiable data: SOS-inducing potency. In comparison to the traditional Ames test, this method possesses a number of advantages, including simplicity, ease of performance, the requirement of a single strain, faster results, and higher sensitivity. In addition, this test does not require the survival of the tester strain, as a result of which even highly toxic substances can be evaluated for genotoxicity. Additional systems using fusion between sulA (an SOS-inducible gene) and phoA (encoding alkaline phosphatase) have been recently developed. ${ }^{16}$ Being a simple enzyme-based colorimetric assay, the SOS chromotest exhibits potential to be transformed into an HTS assay.

The Ames test, Ara D test, and SOS chromotest form the prokaryotic reporter systems for assessing genotoxicity. In addition to these tests, eukaryotic reporter systems involving yeast have also been developed. The main advantage of eukaryotic systems is closer resemblance to the human system in terms of replication and DNA-repair machinery. In addition, a larger cell nucleus and genome enable the detection of rearrangements and genomic aberrations.

\section{Eukaryotic systems The yeast DEL assay and yeast mitotic gene-conversion assay}

The DEL assay can be considered equivalent to the Ames test wherein the eukaryote yeast is employed as the test organism. The strain is altered at the HIS3 locus, due to interruption by short repeats, and thus detects mutagens by its ability to revert the strain back to $\mathrm{His}^{+}$, either by plating on media lacking histidine or in a microtiter plate format using a colorimetric readout. ${ }^{17}$ The assay has been proven to be very accurate, showing $92 \%$ similarity in results when compared to the Ames result for various chemical compounds. Recent developments include the 96- and 384-well microtiter plate format for this assay, thus enabling HTS of compounds. ${ }^{18}$ Gentronix has developed the GreenScreen GC assay using genetically modified yeast to express GFP as a reporter when the RAD54 DNA-repair gene is expressed. Fluorescence end point is used for assessing genotoxicity, whereas optical absorbance is used to assess cytotoxicity in parallel. The technology has been validated using the Johnson and Johnson compound collection. ${ }^{19}$
On the other hand, the gene-conversion assay is used to detect mitotic crossing over, mitotic gene conversions and reverse mutation by employing heteroallelic (ade2-40/ ade2-119 and trp5-12/trp5-27) and homoallelic (ilv1-92/ ilv1-92) gene loci. The presence of white colonies indicates original heteroallelic condition, whereas the presence of pink or red colonies (formed due to homologous genotypes) indicates the occurrence of mitotic crossover. ${ }^{17}$ The loss of auxotrophy for adenine or tryptophan is indicative of mitotic gene conversion. The major advantage of this assay is the detection of reversion- and repair-associated mechanisms, which in turn increase sensitivity to genotoxicity. This assay is currently included in the Code of Federal Regulations of USA. The only disadvantage of this assay is the inability for automation, as the assay involves screening for colony-color change as an indication for genotoxicity.

Rnr3 and HUG1 are two transcriptional reporter systems that currently exist in yeasts. ${ }^{17}$ An initial assay was developed with rnr3 driving lacZ expression ( $r n r 3-l a c Z$ ). Another variant involves the use of secreted Cypridina luciferase as a reporter (rnr3-luciferase) in a DNA repair-deficient yeast strain. The HUG1 promoter is used to drive expression of GFP. However, in comparison to other methods, these assays still have lower sensitivity, but continuous efforts are involved to decipher and understand the mechanisms involved in DNA-damage responses in yeast. This may serve as the foundation for the discovery of novel molecular markers, similar to those in humans that are indicative of DNA damage and can be employed for HTS.

\section{The comet assay/single-cell gel technique}

The comet assay allows the visualization of DNA damage in eukaryotic cells. ${ }^{20}$ The assay is used to detect single- and double-strand breaks, alkali labile sites, abasic sites, oxidative damage, and cross-linking of DNA with DNA, protein, or drug. The assay relies on the fact that intact DNA retains a highly organized association with matrix proteins in the nucleus, which upon damage is disrupted. Therefore, individual strands of DNA relax and expand out of the cavity into the agarose. The application of an electric field promotes the movement of this DNA toward the positively charged anode. Intact DNA strands are too large and remain in the cavity, whereas the smaller fragments migrate in a given period of time. Therefore, the amount of DNA migration can be utilized to measure the amount of DNA damage in the cell. The overall structure resembles a comet (hence "comet assay"), with a circular head corresponding to the intact DNA that remains in the cavity and a tail of damaged DNA. 
Since initial development, the technique has undergone various changes to improve sensitivity to detect and assess genotoxic agents. The technique possesses a number of advantages in comparison to other assays that are currently employed to assess genotoxicity, including high sensitivity, low cost, short turnaround time, and requirement of a small number of cells and minimal quantities of test substance. In addition, the technique possesses the potential not only to be used as an HTS assay for genotoxicity but also to distinguish between genotoxicity- versus cytotoxicity-induced chromosomal damage and genotoxic versus nongenotoxic carcinogens. However, the assay has not yet been validated globally for reproducibility and reliability on an interlaboratory and intralaboratory scale in comparison to other existing methods. The comet assay has been reportedly used for in vitro as well as in vivo testing, the guidelines for which are based on the current guidelines for genetic toxicity testing by Organisation for Economic Co-operation and Development (OECD). ${ }^{21}$

Commonly used cell lines for in vitro studies include mouse lymphoma L5178Y, CHO, and Chinese hamster lung cell lines, and the standard primary cells include human lymphocytes and rodent hepatocytes. In accordance with the guidelines, the cells in suspension or monolayer culture should be exposed to the test substance, with and without metabolic activation, for a range of 3-6 hours. Furthermore, as DNA damage is associated with cell death, it is ensured that the highest dose tested does not induce excessive cytotoxicity (more than $30 \%$ in comparison to the control) and that cytotoxicity is evaluated concurrently. It is also essential that every experiment includes appropriate controls with and without metabolic activation.

The application of the comet assay to in vivo studies is considered a significant development in genetic toxicology studies. ${ }^{22}$ The main advantage lies in the fact that only a small number of cells are required for analysis, and thus any tissue or organ is amenable to investigation.

Another version of the comet assay is based on the treatment of the liberated DNA present in the agarose gels postlysis. ${ }^{23}$ Since only DNA is exposed in this method, an alteration in DNA migration under these conditions indicates the ability of the test substance to induce DNA damage, independently of cytotoxicity.

Limitations of the comet assay include the inability to detect short-lived primary DNA lesions (due to rapid repair) and the appearance of false-positive results due to mechanisms related to cytotoxicity. Though the comet assay exhibits a number of advantages and very few limitations, more evidence of reliability and reproducibility of results is required before the assay can be critically evaluated for its use as a tool for assessing genotoxicity. ${ }^{24}$

Methods are being developed to transform the comet assay into an HTS assay (96-well format) by introducing variables, including the use of suspension cultures instead of attached cells and automated measurement of ATP levels to assess viability. ${ }^{25}$ Gentronix also offers an alkaline in vitro comet assay using TK6 cells. The standard parameter employed by Gentronix to assess genotoxicity using the comet assay is mean percentage tail intensity. ${ }^{26}$ The methodology involves treating cells with five twofold serial dilutions of test chemicals in a 24-well microplate, harvesting and embedding in agarose, followed by lysis and electrophoresis. The slides are dried, stained, and analyzed microscopically using Comet Assay IV software.

The comet chip, developed by integration of biological and engineering principles, is a single-cell microarray for HTS of DNA damage in human cells based on the principle of single-cell gel electrophoresis, employing a 96-well format to enable parallel processing, reduced sample-to-sample variation, and reduced turnaround time. ${ }^{27}$

\section{The micronucleus test}

The micronucleus (small nucleus) can be defined as a third nucleus comprising a portion of acentric or entire chromosome that could not be carried to the opposite poles during anaphase of mitosis or meiosis. This results in the formation of a daughter cell that lacks either a part or a complete chromosome. Therefore, postcytokinesis, one of the daughter cells is observed to have a large and a small nucleus. The micronucleus test is used as a tool to assess genetic damage, as the number of micronuclei formed directly corresponds to the extent of DNA damage. ${ }^{17}$ The sensitivity of the test has been enhanced through coupling with fluorescence in situ hybridization (FISH), wherein the extent of damage can be clearly visualized. Considered to be one of the most reliable tests in assessing genotoxicity, there are two versions of this assay: the in vivo micronucleus test and the in vitro micronucleus test. The presence of micronuclei is most evident in erythrocytes, but it can also be used with other cell types. The main advantage of using erythrocytes for this assay is the lack of a main nucleus. Therefore, all the micronuclei formed are as a result of chromosomal damage.

The in vivo micronucleus test, one of the standard battery of tests carried out to assess the toxicity of various compounds before approval, evaluates the damage induced by the test substance to the chromosomes or the mitotic apparatus 
of erythroblasts by the analysis of erythrocytes sampled in bone marrow and/or peripheral blood cells of animals, usually rodents. ${ }^{28}$ Visualization of micronuclei is facilitated in these cells because they lack a main nucleus. Induced chromosome damage is indicated by the presence of a high number of micronucleated polychromatic erythrocytes.

The in vitro version uses cultured human or rodent cells. It provides a comprehensive basis for investigating chromosome damage, as it allows the detection of both aneugens and clastogens. The in vitro mammalian cell micronucleus test may employ cultures of cell lines or primary cell cultures. The cells are selected based on a number of factors, including growth characteristics, karyotype stability, chromosome number and diversity, and spontaneous formation of micronuclei. The assay involves exposure of cells to the test substance both in the presence and absence of metabolic activation, following which the cells are allowed to grow for a period sufficient to allow chromosome or spindle damage, resulting in micronuclei formation. Microscopic examination of these cells is carried out to detect micronuclei formation. ${ }^{29}$ Ideally, micronuclei are scored in only those cells that have completed mitosis during or after exposure to the test substance. Scoring is done only in binucleated cells when cultures have been treated with a cytokinesis blocker. However, in the absence of a cytokinesis blocker, the cells analyzed must have undergone cell division during or after exposure to the test substance. It is essential that cell proliferation has occurred in both the control and treated cultures, and the extent of cytotoxicity induced by the test substance is evaluated in parallel.

The EpiDerm ${ }^{\mathrm{TM}}$ 3-D human skin model has been used to establish a novel in vitro human reconstructed skin micronucleus assay that measures the micronucleus induced in dividing basal cell keratinocytes. ${ }^{30}$

In order to allow for faster screening of compounds through in vitro micronucleus assay, Gentronix offers the MicroFlow ${ }^{\circledR}$ flow-cytometry micronucleus test developed by Litron Laboratories. This approach utilizes dual labeling to allow automated enumeration of micronuclei from TK 6 cells by flow cytometry, exposed to a test chemical in the absence or presence of metabolic activation using an S9 fraction. ${ }^{31}$

Cerep offers the automation of in vitro micronucleus assay wherein the cells with micronuclei are scored with the help of proprietary image software from Thermo Fisher Scientific. Cerep conducts the micronucleus test using $\mathrm{CHO}$ $\mathrm{K} 1$ cells. The assay is performed in duplicate, analyzing eight concentrations of a compound at a single time point. Usually, 2,000 binucleated cells are scored per concentration.
A micronucleus is considered valid only if it is located in the cytoplasmic area and is less than $33 \%$ the size of the main nucleus exhibiting similar intensity. ${ }^{10}$ Two dyes are used to detect the cytoplasmic region and the nucleus distinctively. Mitomycin C and cyclophosphamide are used as reference compounds in the absence and presence of metabolic activation for the assay. Cerep in association with Unilever has evaluated and validated its automated in vitro micronucleus assay in $\mathrm{CHO} \mathrm{K} 1$ cells. ${ }^{32}$

\section{The mouse lymphoma assay}

This assay utilizes the $T k$ gene to detect point mutation and chromosomal mutations. ${ }^{33}$ It is the most widely used mammalian cell gene-mutation assay, forms an essential component of genotoxicity-testing requirements for registration of various molecules, and is mandatory for regulatory compliance. L5178Y/Tk ${ }^{+/}$mouse lymphoma cell lines are used to detect mutations based on the ability of a compound to inactivate the $\mathrm{Tk}$ allele located on mouse chromosome 11. The selection of the mutants is based on the use of trifluorothymidine, which inhibits the growth of normal cells while promoting the growth of the mutants. Chemicals that are clastogenic in nature give rise to smaller colonies with a slow growth rate, whereas those inducing only point mutations give rise to bigger colonies with a normal growth rate. However, the frequency of the two colony types is highly variable, and depends on the nature of the compound being assessed for genotoxicity. The Tk mutants are characterized by a combination of molecular and cytogenic analyses to understand the nature and type of mutation. Originally developed by Clive et al, ${ }^{34}$ using soft agar to enumerate mutants, and the technique and protocols have been modified and improved to incorporate liquid medium and 96-well microtiter plates for assessing the frequency of mutation. ${ }^{35}$ One of the drawbacks of this assay includes the high incidence of false positives.

A number of other techniques have been conventionally used to assess the genotoxicity of various molecules. These include the sister-chromatid exchange assay and chromosomal aberration test, both involving microscopic examination to assess DNA damage occurring within the cell. The sister-chromatid assay portrays damage of DNA in terms of ongoing DNA-repair mechanisms, and is used along with the micronucleus test to detect clastogens and aneugens. ${ }^{17}$ On the other hand, the chromosomal aberration test is used to detect structural and numerical aberrations with the help of FISH staining techniques. ${ }^{17}$ Both these methods are helpful to assess toxicity, but are 
labor-intensive, time-consuming, and are not ideal for high throughput screening.

Though a large number of biochemical and cell-based methods are available to assess genotoxicity, the fundamental disadvantage is their lack of resemblance to the human physiological system. Therefore, it becomes crucial to establish systems that can mimic the human/mammalian system at the physiological, histological, cellular, and molecular level. However, the establishment of such a system is accompanied by its own set of challenges related to ethical and regulatory domains. In addition, due to the invasive nature of technique that would be involved, the entire exercise would become a labor-intensive and time-consuming affair. As a result, the establishment of a complex system closest to a human system may not really be a suitable option to cater to the requirement of HTS for genotoxicity. It may be a better option to establish simpler systems that possess genetic resemblance to mammalian systems rather than physiological and histological resemblance.

\section{Animal models}

Having a close resemblance to humans, mice serve as the first option. Various transgenic mouse models, such as Muta $^{\text {TM} M o u s e, ~ L a c Z ~ P l a s m i d ~ M o u s e, ~ a n d ~ t h e ~ B i g ~ B l u e ~}{ }^{\circledR}$ assay, have been developed for assessing genotoxicity. ${ }^{17}$ However, mouse models do not have the potential to be transformed into HTS tools, mainly due to their time-, labor-, and resource-intensive nature in comparison to other alternative models.

Zebrafish have been shown to share $70 \%$ sequence homology with humans and possess about $84 \%$ of the same genes as humans that are associated with disease. ${ }^{36}$ Therefore, these organisms may serve as models to assess genotoxicity. Their short generation time with well-characterized developmental stages and similar genetic morphology for humans makes them ideal candidates in the race for the development of in vivo models. Other advantages of using zebrafish as a model system include ease of breeding, low-cost maintenance, smallness, permeable and transparent larvae, and development of larvae outside the host, which would allow the use of noninvasive imaging technology and also reduce the amount of chemicals required for testing. Although not approved by the US Food and Drug Administration (FDA) as a model for higher mammals, zebrafish have been granted limited regulatory approval. The OECD and European Directive 2010/63/ EU have laid the guidelines and approved the use of zebrafish for toxicity and efficacy studies. ${ }^{37}$ The National Center for Toxicological Research has also set up a zebrafish facility to study multiple forms of toxicity. Zebrafish have been used to study hepatotoxicity, nephrotoxicity, cardiotoxicity, and developmental toxicity. ${ }^{5}$ Therefore, in addition to being a well-established in vitro model to assess genotoxicity, it poses as a frontrunner for an emerging in vivo model for studying toxicity.

Due to its well-documented genetics and developmental biology, Drosophila melanogaster has often served as an insect model for the study of human diseases and toxicological research. Recent developments include its use for assessing somatic cell genotoxicity through the wing-spot test by somatic mutation and recombination $w / w^{+}$eye assay. Gross chromosomal changes have also been studied in neuroblast cells of Drosophila. ${ }^{38}$ The comet assay has been recently adapted to assess in vivo genotoxicity in D. melanogaster. ${ }^{38}$ Drosophila has been recommended by the European Centre for the Validation of Alternative Methods as an appropriate model for research and testing. Advantages of using Drosophila as a model organism for assessing genotoxicity include complementarity to bacteria- or yeast-based models, it is extremely cheap and easy to maintain, it produces large numbers of testable offspring, and has metabolic and DNA-repair systems that are highly homologous with human systems. Though assays for genetic damage in germ cells were developed first using Drosophila, recombination assays in somatic cells have also been developed for improved sensitivity. The large number of molecular targets, the suitability for early exposure, and its active metabolism make Drosophila possibly the most sensitive phenotypic detection model available and a very promising model for detection of genotoxic and teratogenic effects.

Caenorhabditis elegans is another model organism that is being considered for assessing genotoxicity. ${ }^{39}$ Phase I of the of National Toxicity Program's Tox 21 research involved testing a large number of compounds using C. elegans in the Worm Tox Lab. ${ }^{40}$

\section{Biomarkers as genotoxicity evaluators}

There is no doubt about the applicability of prokaryotic systems, eukaryotic systems, and whole-animal systems in assessing the genotoxicity of various molecules. However, there is an absolute need for the development of systems that are capable of analyzing the dose responsiveness of multiple compounds at a single time point, or multiple time points in real time, resulting in the availability of quantitative data in short intervals of time. Various strategies of achieving the same involve the development of various 
mammalian cell-based systems with the help of microarrays and next-generation sequencing for identifying new targets and understanding key events that ultimately result in DNA damage. This would serve both the purposes of assessing genotoxicity and understanding the mechanism of action at the molecular level.

Miniaturized three-dimensional cell-culture arrays or the data toxicology-assay chip have taken the screening of various compounds to an all new level, allowing the assessment of toxicity of the compound, as well as its metabolites. This technology encapsulates human cells in three-dimensional hydrogel matrices like collagen or alginate on a glass slide in specific spatial arrangements, such that multiple compounds can be screened simultaneously along with the appropriate controls. The data chip also provides a provision for incubation in relevant media in case the cells need to be grown over extended periods of time. Initiated at a level of 560 spots per slide, the technological advancement now permits 1,080 spots on a single glass slide. ${ }^{41}$ However, to date this has only been used to assess the cytotoxicity of drug candidates and has not yet been adapted for genotoxic studies. A similar technology that has not yet been adapted to assess genotoxicity but with high potential is the microfluidic sensor cell-based chip. ${ }^{42}$ The development of these high-throughput miniaturized cell-based assays minimizes the consumption of cells and reagents, in addition to providing similar in vivo conditions.

Agilent Technologies has developed a microarray-based method to detect and analyze DNA-damage and -repair mechanisms across the genome. The technology is patented, and measures the level of genotoxin-induced damage with the help of immunoprecipitation on a microarray chip. Change in acetylation levels of H3 histone lysine 14 is used to assess genotoxicity. This is compared to changes that have been induced by ultraviolet light. The damaged DNA is affinity-captured by immunoprecipitation, and amplified and labeled using different fluorophores. These are then allowed to hybridize to probes present on a microarray plate, leading to development of signals that are then converted into numerical values. ${ }^{43}$

Cell Ciphr, developed by Cellumen Inc., combines various aspects of genotoxicity assessment, including tissue-specific cells, multiplexed functional biomarkers, and a compound reference library, into a single cell-based assay that allows the analysis of more than ten biomarkers at a single stretch. The assay provides insights into mechanism of action and evaluates toxicity, thereby allowing accurate identification of potential drug candidates. ${ }^{44}$
Thermo Fisher Scientific also offers CellSensor ${ }^{\circledR}$ cell lines coupled with GeneBLAzer ${ }^{\circledR}$ technology to analyze various pathways. ${ }^{45}$ One of the most common cell lines used is the CellSensor p53RE-bla HCT-116, which includes a $\beta$-lactamase gene under the control of a p53 response element, a major element that is involved in DNA repair.

SA Biosciences has introduced the Oligo GEArray ${ }^{\circledR}$ human DNA damage signaling pathway microarray, which screens approximately 113 genes that are known to be involved in DNA damage-response pathways. A polymerase chain reaction (PCR) format of this assay has also been developed that screens approximately 84 genes. The PCR format uses real-time PCR to evaluate the expression of various genes involved in DNA-damage signalling. ${ }^{46}$

Gentronix genotoxicity screens utilize a $G A D D 45 A$ promoter in human cell lines for detecting genotoxic stress. This gene is induced upon exposure to clastogens, aneugens, and mutagens, thereby leading to overexpression under genotoxic conditions. ${ }^{19}$ The GreenScreen HC Assay uses the humanderived p53-competent TK6 cell line with a patented GFPreporter system that exploits the regulation of the GADD45A gene. The patented GFP fluorescence reporter comprises complex regulatory elements that recognize mutagens, aneugens, clastogens, and topoisomerase and polymerase inhibitors. Exposure to genotoxic substances increases the expression of GFP due to overexpression of the GADD45A gene. On the other hand, the assay provides negative results for nongenotoxins that might have been reported otherwise in other in vitro analysis. Therefore, the assay is highly specific and sensitive to detect genotoxic compounds and has undergone extensive validation. A 96-well plate version has been introduced by Gentronix that enables testing four compounds over nine serial dilutions simultaneously. Validations have also been completed for screening of twelve compounds over three serial dilutions in the 96-well format. However, the protocol is still considered to be resource- and labor-intensive. Also, compounds having the ability to autofluoresce cause a lot of interference in the detection of GFP, thereby inculcating the need for a parallel test using a control cell line possessing a GFP-reporter system without GAD45-promoter control. In addition, the data generated through GreenScreen $\mathrm{HC}$ have to be analyzed manually.

A variant of this assay involves the use of a Gaussia luciferase-reporter system instead of the GFP-reporter system. ${ }^{47}$ Gaussia luciferases catalyze the oxidative carboxylation of coelenterazine, resulting in photon generation that can be captured by a luminescence plate reader. This is the BlueScreen HC assay, and has been adapted and automated 
for a 384-well format. The BlueScreen HC is reported to have reduced interference from autofluorescent compounds and easier data analysis, due to complete automation and integration with high-throughput compound-profiling assays. Therefore, Gentronix genotoxicity assays satisfy various criteria of being high-throughput and highly sensitive and specific to enable the assessment of various molecules for genotoxicity, thereby concentrating the efforts to channel nongenotoxic molecules into drug-discovery research. Future aspirations of Gentronix involve transformation from 96-well plates to 384 and 1,536 wells to enable rapid screening of compounds in short periods of time.

HTS using ATAD5 luciferase has been developed, as the ATAD5 protein is stabilized post-DNA damage. The assay has been reported to be highly specific, reliable, and reproducible in a 1,536-well plate format. ${ }^{48}$

The Anthem Signature Genotoxicity Screen is a novel HTS technique using the p53-proficient human HCT116 cell line, developed at Anthem Biosciences. ${ }^{49}$ The assay employs engineered cell clones expressing three reporter genes under transcriptional control of promoters of DNA damage-inducible genes. The three reporter genes include Renilla luciferase, firefly luciferase, and $\beta$-galactosidase, which are fused to promoter regions of p21, GADD153, and p53 respectively. In addition to screening compounds for genotoxicity, this assay also provides an insight into the mechanism of action of genotoxic compounds, thus aiding in rational drug design. This assay has been shown to have very high concordance with in vivo data.

\section{Conclusion}

With a multitude of molecules being introduced into the market in various forms, there is undoubtedly a need for assessing the biosafety of these molecules before their release. Prokaryotic and eukaryotic models have proven to be extremely useful and complement each other. While prokaryotic systems are inexpensive and have the potential to be incorporated into high-throughput format, their applicability is limited to detection of frame shift and point mutations. On the other hand, eukaryotic systems exhibit more sensitivity toward DNA damage, but unfortunately pose the challenge of being time-consuming and labor-intensive. The major challenge of assessing the genotoxicity of compounds lies in the development of systems that are high-throughput, rapid, non-labor-intensive, and cost-effective, yet accurately mimic the human environment. In addition, identifying and characterizing various signaling pathways that are activated during
DNA damage may hold the key to understanding what makes a molecule toxic or nontoxic, thereby aiding in optimizing procedures for production of molecules having high potency at the desired target and minimizing undesirable genotoxicity. Though a number of systems have been developed to assess genotoxicity, the variability in the guidelines for assessing genotoxicity among various countries is a cause for concern. It is probably best to standardize a battery of tests covering different targets and end points to ensure the identification of the majority of genotoxic compounds. With the development of new tools and methodologies to evaluate the genotoxicity of compounds intended for enhancing quality of life, there would be a need to incorporate the same into the regulatory process, thereby ensuring complete safety of the molecule at various levels.

In addition to all of this, the HTS initiative developed by National Toxicity Program aims at evolving toxicological studies from being a mere observatory science to a predictive science with the aid of technology to identify important targets in cellular pathways that play crucial roles in the development of genotoxicity and carcinogenicity. ${ }^{40}$ It lays an emphasis on the development of in vitro biochemical and cell-based assays and envisions the automation of same, so as to provide HTS of various molecules in addition to characterizing toxicity pathways for better understanding of mechanisms of action. The Tox 21 community is the powerhouse of the initiative, and its members include the National Institute of Environmental Health Sciences, the National Institutes of Health/National Chemical Genomics Centre, the Environmental Protection Agency, and the FDA. ToxCast, launched in 2007, uses a combination of computers, genomics, and cellular biology to accelerate toxicity testing, thereby enhancing the capacity for screening new compounds.

\section{Disclosure}

The authors have no conflicts of interest in this work.

\section{References}

1. US Food and Drug Administration. International Conference on Harmonisation; guidance on S2(R1) Genotoxicity Testing and Data Interpretation for Pharmaceuticals Intended for Human Use; availability. Notice. Fed Regist. 2012;77(110):33748-33749.

2. Bhuyan BK, Zimmer DM, Mazurek JH, et al. Comparative genotoxicity of adriamycin and menogarol, two anthracycline antitumor agents. Cancer Res. 1983;43(11):5293-5297.

3. Brambilla G, Martelli A. Genotoxicity and carcinogenicity studies of analgesics, anti-inflammatory drugs and antipyretics. Pharmacol Res. 2009;60(1):1-17.

4. Brambilla G, Mattioli F, Robbiano L, Martelli A. Studies on genotoxicity and carcinogenicity of antibacterial, antiviral, antimalarial and antifungal drugs. Mutagenesis. 2012;27(4):387-413. 
5. Chakravarthy S, Sadagopan S, Nair A, Sukumaran SK. Zebrafish as an in vivo high-throughput model for genotoxicity. Zebrafish. 2014;11(2): 154-166.

6. Ames BN, Lee FD, Durston WE. An improved bacterial test system for the detection and classification of mutagens and carcinogens. Proc Natl Acad Sci U SA. 1973;70(3):782-786.

7. Ames BN, Durston WE, Yamasaki E, Lee FD. Carcinogens are mutagens: a simple test system combining liver homogenates for activation and bacteria for detection. Proc Natl Acad Sci U S A. 1973; 70(8):2281-2285.

8. McMahon RE, Cline JC, Thompson CZ. Assay of 855 test chemicals in ten tester strains using a new modification of the Ames test for bacterial mutagens. Cancer Res. 1979;39(3):682-693.

9. Bridges BA. The fluctuation test. Arch Toxicol. 1980;46(1-2):41-44.

10. Cerep. Genotoxicity. 2013. Available from: http://www.cerep.fr/cerep/ users/pages/Downloads/Documents/Marketing/Pharmacology\%20 $\& \% 20$ ADME/Application\%20notes/Genotoxicity.pdf. Accessed November 4, 2015.

11. Gentronix. Ames MPF. Available from: http://www.gentronix.co.uk/ product/ames-mpf. Accessed November 4, 2015.

12. Guadaño A, de la Peña E, González-Coloma A, Alvarez JF. Development of a new bioluminescent mutagenicity assay based on the Ames test. Mutagenesis. 1999;14(4):411-415.

13. Aubrecht J, Osowski JJ, Persaud P, et al. Bioluminescent Salmonella reverse mutation assay: a screen for detecting mutagenicity with high throughput attributes. Mutagenesis. 2007;22(5):335-342.

14. Ruiz-Vázquez R, Pueyo C, Cerdá-Olmedo E. A mutagen assay detecting forward mutations in an arabinose-sensitive strain of Salmonella typhimurium. Mutat Res. 1978;54(2):121-129.

15. Quillardet P, Huisman O, D'Ari R, Hofnung M. SOS chromotest, a direct assay of induction of an SOS function in Escherichia coli K-12 to measure genotoxicity. Proc Natl Acad Sci U SA. 1982;79(19): 5971-5975.

16. Biran A, Ben Yoav H, Yagur-Kroll S, et al. Microbial genotoxicity bioreporters based on sulA activation. Anal Bioanal Chem. 2011; 400(9):3013-3024.

17. Musgrove C, Camps M. Models for detection of genotoxicity in vivo: present and future. In: Mishra DR, editor. Mutagenesis. Rijeka, Croatia: InTech; 2012:31-50

18. Hontzeas N, Hafer K, Schiestl RH. Development of a microtiter plate version of the yeast DEL assay amenable to high-throughput toxicity screening of chemical libraries. Mutat Res. 2007;634(1-2):228-234.

19. Knight AW, Birrell L, Walmsley RM. Development and validation of a higher throughput screening approach to genotoxicity testing using the GADD45a-GFP GreenScreen HC assay. J Biomol Screen. 2009; 14(1):16-30.

20. Tice RR, Agurell E, Anderson D, et al. Single cell gel/comet assay: guidelines for in vitro and in vivo genetic toxicology testing. Environ Mol Mutagen. 2000;35(3):206-221.

21. Burlinson B. The in vitro and in vivo comet assays. Methods Mol Biol. 2012;817:143-163.

22. Organisation for Economic Co-operation and Development. OECD guideline for the testing of chemicals: In vivo mammalian alkaline comet assay. 2014. Available from: http://www.oecd-ilibrary.org/ docserver/download/9714511e.pdf?expires $=1446582965 \& \mathrm{id}=\mathrm{id} \& \mathrm{acc}$ name $=$ guest\&checksum=E9C03B8F9287629EB00C9CA8C5EF2643. Accessed November 4, 2015.

23. Liao W, McNutt MA, Zhu WG. The comet assay: a sensitive method for detecting DNA damage in individual cells. Methods. 2009;48(1):46-53.

24. Brendler-Schwaab S, Hartmann A, Pfuhler S, Speit G. The in vivo comet assay: use and status in genotoxicity testing. Mutagenesis. 2005;20(4): 245-254.

25. Kiskinis E, Suter W, Hartmann A. High throughput comet assay using 96-well plates. Mutagenesis. 2002;17(1):37-43.

26. Gentronix. Comet assay. Available from: http://www.gentronix.co.uk/ product/comet-assay. Accessed November 4, 2015.
27. Ge J, Prasongtanakij S, Wood DK, et al. CometChip: a high-throughput 96-well platform for measuring DNA damage in microarrayed human cells. J Vis Exp. 2012;(92):e50607.

28. Organisation for Economic Co-operation and Development. OECD guideline for testing of chemicals: Mammalian erythrocyte micronucleus test. 1997. Available from: http://www.oecd.org/ chemicalsafety/risk-assessment/1948442.pdf. Accessed November 4, 2015.

29. Organisation for Economic Co-operation and Development. OECD guidelines for testing of chemicals: Proposal for updating test guideline 487 - In vitro mammalian cell micronucleus test. 2012. Available from: http://www.oecd.org/env/ehs/testing/TG487\%20Oct\%202012\%20 updated\%2029oct.pdf. Accessed November 4, 2015.

30. Kirsch-Volders M, Decordier I, Elhajouji A, Plas G, Aardema MJ, Fenech $\mathrm{M}$. In vitro genotoxicity testing using the micronucleus assay in cell lines, human lymphocytes and 3D human skin models. Mutagenesis. 2011;26(1):177-184.

31. Gentronix. In vitro mammalian cell micronucleus test (OECD 487). Available from: http://www.gentronix.co.uk/product/micronucleus-test. Accessed November 4, 2015.

32. Diaz D, Scott A, Carmichael P, Shi W, Costales C. Evaluation of an automated in vitro micronucleus assay in CHO-K1 cells. Mutat Res. 2007;630(1-2):1-13.

33. Lloyd M, Kidd D. The mouse lymphoma assay. Methods Mol Biol. 2012;817:35-54

34. Clive D, Johnson KO, Spector JF, Batson AG, Brown NM. Validation and characterization of the L5178Y/TK+/- mouse lymphoma mutagen assay system. Mutat Res. 1979;59(1):61-108.

35. Honma M, Hayashi M, Shimada H, et al. Evaluation of the mouse lymphoma TK assay (microwell method) as an alternative to the in vitro chromosomal aberration test. Mutagenesis. 1999;14(1):5-22.

36. Howe K, Clark MD, Torroja CF, et al. The zebrafish reference genome sequence and its relationship to the human genome. Nature. 2013;496(7446):498-503.

37. [No authors listed]. Directive 2010/63/EU of the European Parliament and the Council of 22 September 2010 on the protection of animals used for scientific purposes. OJEU. 2010;276:33-79.

38. Siddique HR, Chowdhuri DK, Saxena DK, Dhawan A. Validation of Drosophila melanogaster as an in vivo model for genotoxicity assessment using modified alkaline comet assay. Mutagenesis. 2005; 20(4):285-290.

39. Meier B, Gartner A. Having a direct look: analysis of DNA damage and repair mechanisms by next generation sequencing. Exp Cell Res. 2014;329(1):35-41.

40. National Toxicology Program. Tox21. Available from: http://ntp.niehs. nih.gov/results/hts/index.html. Accessed November 4, 2015.

41. Lee MY, Kumar RA, Sukumaran SM, Hogg MG, Clark DS, Dordick JS. Three-dimensional cellular microarray for highthroughput toxicology assays. Proc Natl Acad Sci U SA. 2008;105(1): 59-63.

42. Fernandes TG, Diogo MM, Clark DS, Dordick JS, Cabral JM. High-throughput cellular microarray platforms: applications in drug discovery, toxicology and stem cell research. Trends Biotechnol. 2009;27(6):342-349.

43. Powell JR, Bennett MR, Evans KE, et al. 3D-DIP-Chip: a microarraybased method to measure genomic DNA damage. Sci Rep. 2015; 5:7975.

44. High Content Toxicology: CellCiphr ${ }^{\circledR}$ Premier. Available from: http:// www.cyprotex.com/toxicology/multiparametric/cellciphr-premier/. Accessed November 4, 2015.

45. Thermo Fisher Scientific. CellSensor ${ }^{\circledR}$ p53RE-bla HCT-116 cell line. Available from: https://www.thermofisher.com/order/catalog/product/ K1640. Accessed November 4, 2015.

46. SABiosciences. Oligo GEArray ${ }^{\circledR}$ human DNA damage signaling pathway microarray. Available from: http://saweb2.sabiosciences.com/gene_array _ product/HTML/OHS-029.html. Accessed November 4, 2015. 
47. Simpson K, Bevan N, Hastwell P, et al. The BlueScreen-384 assay as an indicator of genotoxic hazard potential in early-stage drug discovery. J Biomol Screen. 2013;18(4):441-452.

48. Fox JT, Myung K. Cell-based high-throughput screens for the discovery of chemotherapeutic agents. Oncotarget. 2012;3(5):581-585.
49. Rajakrishna L, Unni SK, Subbiah M, et al. Validation of a human cell based high-throughput genotoxicity assay 'Anthem's Genotoxicity screen' using ECVAM recommended lists of genotoxic and nongenotoxic chemicals. Toxicol In Vitro. 2014;28(1):46-53.

\section{Publish your work in this journal}

International Journal of High Throughput Screening is an international, peer-reviewed, open access journal publishing original research, reports, editorials, reviews and commentaries dedicated to all aspects of high throughput screening, especially related to drug discovery and associated areas of biology and chemistry. The manuscript management sys-

tem is completely online and includes a very quick and fair peer-review system. Visit http://www.dovepress.com/testimonials.php to read real quotes from published authors.

Submit your manuscript here: http://www.dovepress.com/international-journal-of-high-throughput-screening-journal 